\title{
The Case for a Planetary Defense-Optimized NEO Characterization Tour
}

Lead Author: Andrew S. Rivkin ${ }^{1}$ (andy.rivkin@jhuapl.edu, 443-778-2811)

Co-Authors and Co-Signers: R. Terik Daly ${ }^{1}$, Justin Atchison ${ }^{1}$, Brent Barbee ${ }^{2}$, Paul Abell ${ }^{3}$, Angela Stickle ${ }^{1}$, Thomas S. Sotirelis ${ }^{1}$, Patrick K. King ${ }^{1}$, Derek Richardson ${ }^{4}$, Benjamin Greenhagen $^{1}$, Olivier Barnouin ${ }^{1}$, Cristina Thomas ${ }^{5}$, Dawn Graninger ${ }^{1}$, Carolyn Ernst ${ }^{1}$, Stefanie N. Milam², Daniel J. Scheeres ${ }^{6}$

1. Johns Hopkins University Applied Physics Laboratory

2. NASA Goddard Space Flight Center

3. NASA Johnson Space Center

4. University of Maryland

5. Northern Arizona University

6. University of Colorado 


\section{Introduction}

For the first time, planetary defense priorities are being included in the community's decadal re-evaluation of planetary science. Several contributions from the planetary defense community to the first round of white papers contain some mention or discussion of reconnaissance missions for characterizing Potentially-Hazardous Asteroids (PHAs) [1-7]. In this paper we will consider what such missions might best accomplish, and which general objectives might not be easily reconcilable. We will consider the benefits and drawbacks of the asteroid Apophis as a target for a characterization mission, include a set of recommendations for future characterization missions, and suggest studying a "Potentially-Hazardous Asteroid Characterization Tour" (PHACT) mission concept optimized for planetary defense.

\section{The importance of characterization}

Those aspects of planetary defense that don't deal with policy or law are typically divided into detection, characterization, and mitigation. Detection is, necessarily, the top priority of the planetary defense community. Accordingly, there is a widespread consensus, frequently expressed by the Small Bodies Assessment Group (SBAG) that a space-based survey telescope like the NEO Survey Mission (NEOSM) is the highest priority mission for planetary defense. This capability would be in addition to maintaining ongoing or imminent ground-based survey efforts (e.g., Vera Rubin Observatory).

Mitigation techniques are also represented in NASA's current mission portfolio, with the Double Asteroid Redirection Test (DART) scheduled for launch in 2021 and impact into Dimorphos in 2022, testing the kinetic impactor technique. What has been noticeably absent thus far are plans for characterizing asteroids specifically cognizant of the unique priorities and needs of the planetary defense community, save for support of groundbased spectral and photometric surveys through the R\&A program.

Miller et al. [8] argued in "Asteroid Characterization Priorities for Planetary Defense" that the characteristics needed to mitigate a specific impact threat are: orbit, mass, composition, porosity, shape, structure, and spin, in descending order of importance (Table 1). Subsequent work related to the DART impact shows that the block fraction and size-frequency distribution (SFD) could have a strong influence on the results of a kinetic impact deflection ([9-11], also in Table 1). Barbee provided a similar (but not identical) list at the January 2020 SBAG meeting ${ }^{1}$, again focused on the characterization that would be required for a specific impact threat. Abell et al. (2020) looked in detail at several levels of characterization, with broadly similar results to earlier findings, but also adding the presence/absence of satellites [4].

Here, we instead look at characterization that can be done in advance of an emergency. It is important to keep in mind that difference when comparing our recommendations to the content of the Miller et al. and Barbee work. For the remainder of this paper, we will adopt the Miller et al. prioritized ranking (with block fraction and presence/absence of satellites appended), noting that priorities within this list may evolve but should be comprehensive. Some items can be measured or deduced from Earth-based optical or radar observations, though with limited accuracy or only as hemispherical averages. Many others require a spacecraft visit.

1

https://www.Ipi.usra.edu/sbag/meetings/jan2020/presentations/Barbee_NEO Characterization PD SBAG_202001-16.pdf 


\begin{tabular}{|c|c|c|c|c|}
\hline Property & Earth-based & Flyby & Rendezvous & Note \\
\hline $\begin{array}{l}\text { Orbit: Impact } \\
\text { trajectory or } \\
\text { miss? }\end{array}$ & $\begin{array}{l}\text { Telescopic } \\
\text { measurements } \\
\text { typical, augmented } \\
\text { by radar }\end{array}$ & $\begin{array}{l}\text { May be useful where } \\
\text { groundbased observing } \\
\text { is difficult and impact } \\
\text { probability } \gg 0\end{array}$ & $\begin{array}{l}\text { Provides precise } \\
\text { position, opportunity for } \\
\text { monitoring }\end{array}$ & $\begin{array}{l}\text { Already need good } \\
\text { orbit from Earth- } \\
\text { based data to } \\
\text { allow visit. }\end{array}$ \\
\hline Mass & $\begin{array}{l}\text { Objects with } \\
\text { satellites, else } \\
\text { estimated from } \\
\text { composition and size }\end{array}$ & $\begin{array}{l}\text { Not measurable } \lesssim 3 \mathrm{~km} \\
\text { with current techniques. } \\
\text { Concepts exist for } \\
\text { bodies }<1 \mathrm{~km}[20]\end{array}$ & $\begin{array}{l}\text { Expected uncertainty } \\
1.6 \% \text { for } 160 \text {-m object } \\
{[22]}\end{array}$ & $\begin{array}{l}\text { Density variations } \\
\text { of } \sim 1-5 \% \text { exist } \\
\text { within same-group } \\
\text { meteorite falls }\end{array}$ \\
\hline $\begin{array}{l}\text { Composition: } \\
\text { rock vs. ice vs. } \\
\text { metal }\end{array}$ & $\begin{array}{l}\text { If sufficiently bright } \\
(\mathrm{V}<18), \text { can identify } \\
\text { meteorite analogs }\end{array}$ & $\begin{array}{l}\text { Can identify meteorite } \\
\text { analogs, obtain } \\
\text { resolved data }\end{array}$ & $\begin{array}{l}\text { Can identify meteorite } \\
\text { analogs, obtain resolved } \\
\text { data }\end{array}$ & $\begin{array}{l}\text { Spectrometer } \\
\text { preferred, can also } \\
\text { use filters/imaging }\end{array}$ \\
\hline Porosity & & $\begin{array}{l}\text { Limited by mass/density } \\
\text { uncertainties }\end{array}$ & $\begin{array}{l}\text { Limited by density } \\
\text { variations in materials }\end{array}$ & See mass note \\
\hline $\begin{array}{l}\text { Shape: local } \\
\text { slopes }\end{array}$ & $\begin{array}{l}\text { From lightcurve } \\
\text { and/or radar } \\
\text { measurements }\end{array}$ & $\begin{array}{l}\text { Volume uncertainty } \\
<20 \% \text {, from lightcurves } \\
+ \text { in situ images. [23] }\end{array}$ & $\begin{array}{l}\text { Volume uncertainty } \\
<0.2 \% \text { (Bennu). Shape } \\
\text { accurate to } 0.1-1 \mathrm{~m} .[24]\end{array}$ & $\begin{array}{l}\text { Measured from } \\
\text { stereo imaging, } \\
\text { lidar. }\end{array}$ \\
\hline $\begin{array}{l}\text { Structure: } \\
\text { solid vs. } \\
\text { rubble pile }\end{array}$ & $\begin{array}{l}\text { Inferred from rotation } \\
\text { period, radar } \\
\text { imagery }\end{array}$ & $\begin{array}{l}\text { Inferred from geology, } \\
\text { density estimates }\end{array}$ & $\begin{array}{l}\text { Inferred from geology, } \\
\text { density; radar } \\
\text { tomography possible }\end{array}$ & $\begin{array}{l}\text { Landed } \\
\text { investigations not } \\
\text { considered } \\
\end{array}$ \\
\hline $\begin{array}{l}\text { Spin period, } \\
\text { pole }\end{array}$ & $\begin{array}{l}\text { High precision via } \\
\text { lightcurves }\end{array}$ & $\begin{array}{l}\text { Improved pole position } \\
\text { possible }\end{array}$ & $\begin{array}{l}\text { Further improved pole } \\
\text { position, period }\end{array}$ & \\
\hline $\begin{array}{l}\text { Block fraction, } \\
\text { SFD }\end{array}$ & $\begin{array}{l}\text { Infer from thermal } \\
\text { inertia, but } \\
\text { interpretations wrong } \\
\text { for Bennu/Ryugu }\end{array}$ & $\begin{array}{l}\text { Measured to resolution } \\
\text { limit for lit fraction of } \\
\text { surface }\end{array}$ & $\begin{array}{l}\text { Measured to resolution } \\
\text { limit for lit fraction of } \\
\text { surface }\end{array}$ & $\begin{array}{l}\text { Want block SFD to } \\
\text { scale of kinetic } \\
\text { impactor ( m-size) }\end{array}$ \\
\hline Multiplicity & $\begin{array}{l}\text { Lightcurve and/or } \\
\text { radar }\end{array}$ & $\begin{array}{l}\text { Ida/Dactyl is type case } \\
\text { of satellite detection } \\
\text { during flyby. }\end{array}$ & $\begin{array}{l}\text { Satellites can be } \\
\text { detected, orbits } \\
\text { determined }\end{array}$ & \\
\hline
\end{tabular}

Table 1: Important asteroid characterization properties for planetary defense. Also included is how well each property is measurable from groundbased, flyby, or rendezvous approaches.

Absent a specific impact threat, collecting characterization data from the population at large provides the range of possibilities and an estimate of the frequency of conditions and processes relevant to planetary defense present in the PHAs. This information would be valuable for better understanding the NEO hazard and provides crucial information for planning mitigation capabilities and techniques, should the need arise. In the event of short warning times, in-situ reconnaissance of the actual threat may not be possible prior to launching a mitigation mission, and understanding the range of what might be encountered narrows the design space. It also allows for more realistic, probability-based scenario planning, and better-informed policy making. At this writing, only three objects relevant to planetary defense (in terms of size and orbit) have been encountered by spacecraft: Itokawa, Ryugu, and Bennu (Figure 1, by Emily Lakdawalla). We expect the members of the Didymos-Dimorphos system to be added to this list in the coming decade thanks to DART and Hera, and the Janus smallsat mission will target two NEO binaries (though many details have not yet been made public). Other asteroidal targets, from Ceres and Vesta to Steins and Toutatis pose no threat to Earth, are much larger in diameter than most likely impactors $(<200 \mathrm{~m})$, and have been imaged too coarsely to provide information 

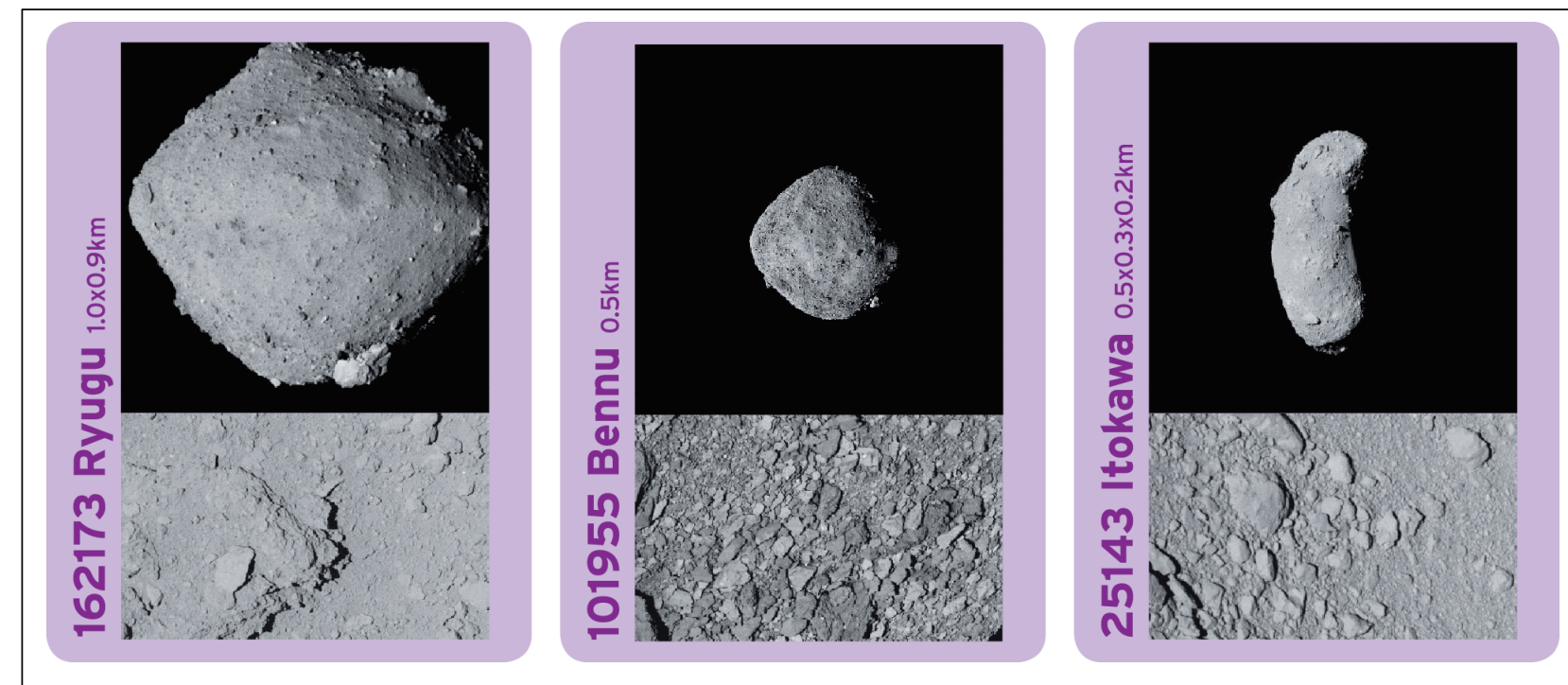

Figure 1: Each image inset is 60 meters wide. By Emily Lakdawalla, using data from JAXA/ISAS (Ryugu, Itokawa) and NASA/JPL/U. Arizona (Bennu)

of particular use for planetary defense. Despite the success of these missions, the population of well-characterized, planetary defense relevant PHAs represents a tiny sample of possibilities that does not cover our already-existing knowledge of the extent of asteroidal variation.

\section{Apophis as a planetary defense target}

The upcoming close pass of Apophis in 2029 provides an interesting opportunity for a spacecraft visit. Such a mission has been advocated by several authors, and has been studied by NASA as well as via a student project $[12,13]$. Given this, it is natural to investigate how a mission to Apophis might be used to address the highest priorities of both the science and planetary defense communities. However, it is not obvious how best to do so.

The goals of a characterization mission oriented toward either planetary defense or planetary science will have overlapping but not identical priorities. This is particularly true in the case of a mission to Apophis. For instance, one of the more interesting phenomena that an Apophis mission might seek to measure is whether the tidal forces experienced by Apophis during its close approach will be sufficient to lead to regolith overturn and a reset of space weathering effects [7]. As important as that question is for asteroid science, however, it is not obvious that it (or any of its implications) are relevant for planetary defense. To the extent that this question can be answered from data that would be collected for planetary defense purposes, it would be a welcome ancillary benefit. To the extent that it drives operations or payload selections, it might serve as a distraction from core planetary defense mission objectives, and would instead be more appropriate in the competed Discovery program. We echo the recommendation of a recent NASM study that missions meeting high-priority planetary defense objectives should not be required to compete against missions meeting high-priority science objectives.

Apophis might experience measurable seismicity during its close Earth encounter, which could allow interior measurements to be made, and such a measurement is the only one that requires an in situ landed package [5]. However, such measurements could be made with less uncertainty on other objects (or Apophis itself) at other times if a seismic source is brought [14]). While a thorough consideration of a hypothetical Apophis mission is not the goal of this paper, such a mission would likely be most useful if it took advantage of specific investigations that can 
only be done at Apophis. Otherwise, more accessible objects will provide better opportunities for conducting experiments under less time pressure and less concern (whether well-founded or illfounded) for possible unintended consequences on an object with an orbit very close to Earth's.

Obviously, the details of any future planetary defense missions will depend upon conditions that are as-yet uncertain: for instance, whether these missions are competed or directed, and whether possible targets and architectures are to be defined by proposers (like the current Discovery competitions) or by NASA (like the current New Frontiers competitions).

It has also been suggested that a mission could be designed to test how quickly a spacecraft could be built and sent to a target as though it were visiting a PHA on a possible impact trajectory that needed to be characterized. Such a mission, focused on an engineering/management challenge rather than on measurements of asteroids, is of a different sort altogether than what we are considering, and again is concerned with characterization of a specific (hypothetical) threat. It is conceivable that such challenges could be part of the mission concept we describe below, though it would need to be made clear from the outset whether the engineering challenge or asteroid investigation would be the primary driver of the project. Making those trades is out of scope of this white paper. We recommend that if an Apophisfocused rendezvous mission is studied, that the prospect of additional asteroid encounters (either before or after Apophis) also be investigated.

\section{Potentially Hazardous Asteroid Characterization Tour}

We propose that the planetary defense objectives of a mission to Apophis, if one is being considered, can best be conducted during one or more encounters of a multi-encounter Potentially Hazardous Asteroid Characterization Tour (PHACT) and recommend studying such a mission concept as part of this Decadal Survey effort. The PHACT would have four objectives:

1. Increase the sample size of relevant objects that have been studied for planetary defense purposes to characterize the PHA population and its diversity and inform models of impacts and other mitigation techniques.

2. Observe the known PHAs that are most likely to impact Earth in the distant future to improve their prediction models and to act as analogs for as-yet unobserved threats.

3. Use short-range observations to give context to ground-based optical and radar measurements, increasing the utility of existing observatories.

4. Test and demonstrate flyby focused technologies, instruments, and operations to characterize their capabilities and limitations in a planetary defense scenario.

\section{Proactive reconnaissance and context building}

There are no asteroids known to be on an impact trajectory with the Earth. However, the known asteroids can be ranked in order of their impact probability, or other similar metrics that include the consequences of an impact if one occurred, with the JPL Center for NEO Studies keeping such a list at https://cneos.jpl.nasa.gov/sentry/. At this writing, there are 5 objects on this list with impact probabilities $>0.1 \%$ over the next century, all with estimated ${ }^{2}$ diameters $<40 \mathrm{~m}$. The three objects with the largest Palermo Scale values, which is a measure combining impact probability and consequence ${ }^{3}$, are 1950 DA $(\sim 1.3-\mathrm{km}$ diameter and possibly metallic), Bennu,

\footnotetext{
${ }^{2}$ Note that these estimates are based on a $15 \%$ albedo. If any of them have albedos of $4 \%$ like C-complex asteroids, their true diameters would be a factor of $\sim 2$ larger than these estimates.

${ }^{3}$ https://cneos.jpl.nasa.gov/sentry/palermo scale.html
} 
and Apophis. The risk from each is below the general background risk from the population.

Target selection for a PHACT mission could be drawn from a mix of objects with higher Palermo Scale values, higher probabilities of impact, and objects that appear representative of the impactor population. At this writing, 966 NEOs have been detected by radar, and visits to these objects will both provide the opportunity for further ground-truthing of radar measurements and the prospect for utilizing radar data to complement spacecraft data, potentially influencing the payload choices.

The experiences of OSIRIS-REx at Bennu and Hayabusa2 at Ryugu underscore that we have not visited enough asteroids to know what to expect at objects that are yet-unexplored. Of particular relevance to planetary defense, both objects were much blockier and regolith-poor than expected based on their thermal inertia or based on the Hayabusa imagery of Itokawa. In addition, Bennu is emitting particles (possibly ejected by micrometeorite impacts), a condition unforeseen by the community [15]. It is not known whether the ways in which Ryugu and Bennu differ from Itokawa are a matter of composition or a matter of random chance. Itokawa, Bennu, Ryugu, and each of the components of Janus' binary targets are all larger than the most likely impactors: we do not know the nature of $\sim 150$-m (or smaller) objects, and only DART and Hera's visits to Dimorphos are planned to an object of that size (though a possible extended mission for Hayabusa2 could visit one of two 50-m objects in the 2029-2031 timeframe). Targeting smaller objects may be a particular situation where science and planetary defense objectives differ: Visits to objects the size of the Chelyabinsk $(\sim 20 \mathrm{~m})$ or Tunguska $(\sim 50 \mathrm{~m})$ impactors would help us understand the extent to which those impacts were typical or unusual.

\section{The Roles and Opportunities of Flybys}

Flybys are potentially very useful for characterization of potentially-hazardous asteroids. The 2010 report Defending Planet Earth did not favor flybys for NEO characterization relevant for mitigation, considering the data return "low relative to cost" and noting that "gamma-ray, x-ray, and other compositional measurements" were not possible during flybys, finding "Dedicated flyby spacecraft missions to NEOs provide only limited information relevant for hazard mitigation issues". These statements are of limited applicability today for several reasons.

First, it is not likely that the 2010 report considered flyby tours. A tour will obviously provide value beyond a single-object mission. Second, the asteroid flybys that are possible with current instrumentation and navigational techniques far outperform asteroid flybys that occurred in the 1990s, or even the flybys of Steins and Lutetia that occurred in 2008 and 2010. All of these flybys were done with spacecraft that were optimized for rendezvous operations (and except for the Mathilde flyby, none of the spacecraft had asteroids as their primary target), and none obtained data within $800 \mathrm{~km}$ of the asteroid. Image resolutions better than $1 \mathrm{~m} /$ pixel are possible on flybys via existing, mature technology, and will be obtained by the DART kinetic impactor mission [16]. In terms of compositional data, the level of precision needed for mitigation reasons [8] is easily obtainable from astronomical techniques rather than requiring elemental measurements that are only available with rendezvous. All of these advances, in combination with the less-constrained mission design flybys have compared to rendezvous missions, have led to recognition that flybys may provide important initial reconnaissance in actual impact scenarios [4]. We can also return to the Barbee SBAG presentation, which again concerns what might be useful for characterizing a specific impact threat. Even in this case, 9 out of the 10 most important priorities are measured to at least a partial degree on flybys, with the only unmeasured one relating to target-area imaging for actual mitigation. 
More to the overall point, this is not an either/or choice. Flybys have a place in an overall characterization strategy, just as rendezvous, landed packages, and astronomical surveys do. As noted below, trajectories have been identified with dozens of flyby targets in a single mission. Given the pace of operations during small bodies rendezvous missions like Hayabusa, Hayabusa2, and OSIRIS-REx, it is likely that the only way to significantly increase our sample size of explored PHAs on a decadal timescale is via a tour solely or largely consisting of flybys.

\section{Characterization Tour: Description and Trades}

A PHACT mission could potentially take several forms, and relevant mission design work has been carried out by several groups through the years. One approach would be would be to build a single, capable spacecraft designed to conduct the entire tour, similar to the Discoveryclass MANTIS concept [17] or the CASTAWAY concept proposed to ESA [18]. An alternative would be to build several small spacecraft, each sent to a different target, and piece together the components of a tour from individual spacecraft visits. This sort of approach was studied via the Ross (originally called "Caesar") PSDS3 smallsat study ${ }^{4}$. We do not further consider a smallsat or cubesat constellation in this paper, though we recognize that they may provide suitable solutions for some planetary defense characterization priorities.

Proof-of-concept mission design work relevant to a PHACT mission scenario are in the literature, though of course the specific targets and trajectories would differ if such a mission were actually to fly. The " $4^{\text {th }}$ Global Trajectory Optimisation Competition" held in 2009 centered on visiting as many NEOs as possible in 10 years with a spacecraft mass of at least $500 \mathrm{~kg}$, finishing with an NEO rendezvous, using solar-electric propulsion, and not using any gravity assists. Two teams ${ }^{5}$ found trajectories that visited 44 asteroids, and 8 teams found trajectories that visited more than 30 asteroids. Even if a more detailed study using more realistic spacecraft properties reduced these numbers by $50 \%$, the resulting mission would be a boon to planetary defense. All-rendezvous mission designs also exist: Barbee et al. (2009) presented an example trajectory with 6 rendezvous with sub-km asteroids [19]. Anecdotally, mission design for the MANTIS asteroid tour concept [17] found numerous trajectories that included Apophis, and a tour with encounters prior to and after Apophis' 2029 close approach should be feasible.

The question of whether a characterization tour should consist of flybys, rendezvous, or a mix of both could be considered an open trade. Table 1 collects quantitative and qualitative measures of the relative effectiveness of flybys, rendezvous, and Earth-based data for addressing characterization priorities. Mass measurements are more precise when made during rendezvous, but there are instrument concepts that allow useful mass measurements to be made during flybys for sub-km objects [20]. Flyby imaging in visible wavelengths will only cover the hemisphere that is lit at the time of the flyby, while rendezvous imaging can cover an entire rotation. However, an unlucky combination of pole position and season could leave a rendezvous spacecraft unable to image the winter hemisphere, while flybys of sufficiently rapid rotators could image much more than half of the surface.

Relatedly, the payloads chosen would depend on whether flybys, rendezvous, or a mixture are favored for the tour. Because the ejecta from kinetic impactors is affected by topography on the scale of the spacecraft [21], sub-meter imaging resolution is required to determine the block SFD at the appropriate scale. An imager is necessary for that investigation, though given the

\footnotetext{
${ }^{4}$ https://www.hou.usra.edu/meetings/smallsat2018/pdf/12 Clark.pdf

${ }^{5}$ https://sophia.estec.esa.int/gtoc portal/wp-content/uploads/2014/11/gtoc4 summary of results.pdf
} 
imaging scale and the size of likely target objects, a wide-angle camera may not be necessary. Simulations suggest that DART will be able to measure the volume of Dimorphos to better than $35 \%$ via imagery, suggesting a flyby that images both incoming and outgoing hemispheres should have uncertainties of less than 20\% [24]. Higher-precision measurements of mass and volume would be possible for many objects from rendezvous imagery, radio science, and lidar measurements, but density variation within meteorite groups sets a limit of $\sim 3-5 \%$ on porosity precision in any case. While sample returns could plausibly (but not necessarily) enable a more precise porosity measurement for a body, it is not obvious that obtaining porosity uncertainties $<5 \%$ are necessary for planetary defense purposes.

The minimum number of objects for a tour is a question without a straightforward answer. Given that six asteroid systems are or will be characterized in the coming decade (Itokawa, Ryugu, Bennu, Didymos-Dimorphos, 1996 FG3, 1991 VH), we suggest a minimum six-system tour since it doubles the amount of data available, but we recognize that shorter tours with particularly high-priority objects may be of equal or greater value.

Recommendations for Studying A Characterization Tour For a Planetary Defense Mission Line

Mainzer et al. (2020) [1] recommended the creation of a NASA Planetary Defense Mission Line funded at $\sim \$ 200-\$ 250 \mathrm{M} /$ year over the next decade to enable missions so that, among other things, "NEO physical properties will be characterized well enough to enable effective mitigation". This should, in principle, allow a cost box to be determined for individual missions in a prospective planetary defense mission line.

We recommend that the Decadal Survey include a study of a PHACT consistent with the funding profile recommended by [1] (as appropriate). We suggest that such a study should consider including Apophis and/or other high priority targets of interest in its tour, as well as at least one object $\mathbf{5 0}$ meters in diameter or smaller, and additional targets to reach what is deemed a sufficient number of encounters. Other possible trades include single vs. multiple spacecraft, including cubesat augmentations of a main spacecraft.

References: [1] Mainzer, A. K. et al. (2020), Planetary Decadal white paper \#293, 15 July 2020; [2] Barbee, B. et al. (2020a) Planetary Decadal white paper \#138, 14 July 2020.; [3] Barbee, B. et al. (2020b), Planetary Decadal white paper \#141, 14 July 2020.; [4] Abell, P.A. et al. Planetary Decadal white paper \#306, 15 July 2020.; [5] Binzel, R.P. et al. (2020) Planetary Decadal white paper \#57, 11 July 2020.; [6] Nolan, M.C. et al. (2020), Planetary Decadal white paper \#251, 15 July 2020.; [7] Cheng, A.F. et al. (2020), Planetary Decadal white paper \#88, 14 July 2020.; [8] Miller, P. et al. (2015). 2015 Planetary Defense Conference IAA-PDC-15-03-01.; [9] Graninger, D. et al. (2019). 2015 Planetary Defense Conference IAA-PDC-19-04-05.; [10] Harrell, J., et al. (2019). AGU Fall Meeting 2019. AGU.; [11] Stickle et al. (2017) Procedia Engineering 204.; [12] MIT Project Apophis (2017). https://eapsweb.mit.edu/mit-project-apophis; [13] Plescia, J. B. et al. (2018) LPI, (2083), 1999.; [14] Anderson, Robert C., et al. LPI 1777 (2014): 1571.; [15] Lauretta, D. S., et al. (2019. Science, 366.; [16] Adams, E, et al. (2019) 2019 IEEE Aerospace Conference.; [17] Rivkin, A. S. et al. (2016) 2016 IEEE Aerospace Conference.; [18] Bowles, N. E. et al. (2018). Adv. Sp. Res., 62.; [19] Barbee, B. W., et al. (2009). Adv. Astron. Sci., 135. [20] Atchison, J. A. (2017). NIAC Swarm Flyby Gravimetry Phase II Report.; [21] Syal, M. B., et al. (2016). Icarus, 269, 50-61.[22] Zannoni et al. (2018). Adv. Sp. Res, 62.; [23] Barnouin et al. (2019) LPSC 2019. [24] Barnouin et al. (2020) Plan. Space. Sci., 180. [25] NASM (2019), https://doi.org/10.17226/25476. 